disturbance, with the presence of cadaverine and putrescine in the urine. These alkaloids are in themselves comparatively innocuous, but he argues that the same organisms which have produced them may also have produced another ptomaine capable of breaking down the red corpuscles. Such a body, however, he did not actually find. The theory is developed at length in connection with a case which he has published in detail 9 but where post mortem very complete atrophy of the gastric mucous membrane was found. It has been known for long that pernicious anæmia is associated with this condition, cases having been described by Fenwick, Brabazon, and many foreign observers, and where such a serious lesion exists it seems hardly necessary to call in the aid of a special microbe or a special poison manufactured by it, to account for the bloodlessness. In such a case as this the intestinal maldigestion, and the unusual putrefactive processes would seem to be immediately referable to the stomach condition, on which the anæmia also probably depends.

On the other hand, a study of the accompanying analyses of the liver shows that pernicious anæmia is not the only condition in which excessive amounts of iron are found in that organ, nor is the quantity of the metal greater in it than in some other diseases. The case of Addison's disease may be disregarded, as we have no knowledge of its influence on the blood; in malaria the corpuscles are destroyed by organisms which lodge in them, and the hroken-down products and iron pigment may be found all over the body, the iron being largely taken up by the liver cells. In the other cases there had been interstitial bleedings, and it is from these, I believe, that the excess of iron found in the livers had been derived.

In those cases of fatal anæmia, where the blood loss was external, very little iron was found in the organs. Further, after artificially-made subcutaneous bleedings in rabbits, and during the absorption of large internal hæmorrhages in man-hæmatocele, scurvy, etc.-iron is plentifully deposited in the liver, and a large amount of yellow pigment is formed from the hæmoglobin of the extravasated blood.

These facts seem to bear out my view that the deposit of iron in the liver and elsewhere and the general diffusion of yellow pigment are not peculiar to pernicious anæmia, but occur in consequence of the interstitial hæmorrhages which take place in some cases of extreme anæmia.

\section{Method OF ANALYsis.}

In conclusion, I may shortly describe my method of analysis, as it may be of interest to those who are working at the subject. One hundred grammes of liver were cut up into small pieces, and the blood carefully washed out with water. It was put into strong alcohol for a few days, pounded in a mortar, and then dried, first on the water bath and afterwards in an air chamber at $105^{\circ} \mathrm{C}$. The loss of water was determined by weighing it. Ten grammes were then gradually added to fused nitrate of potassium in a porcelain capsule kept hot by a Bunsen flame, and when the organic matter was all burned off the iron was left as ferric hydrate. The contents of the capsule were then taken up with hot water, and filtered, the ferric hydrate, which is retained on the filter, being repeatedly washed with water, and then dissolved in dilute sulphuric acid ( 1 to 4 ). As there are always reducing substances present, the solution was heated on the water bath with gradually added quantities of solution of potassium permanganate until reduction no longer took place. If an excess of the permanganate solution had been added, or if binoxide of manganese had been formed, these were got rid of by adding sufficient peroxide of hydrogen solution, the excess of this being driven off by boiling for a few minutes. The solution was then merely tinged pink with permanganate of potassium, and allowed to stand for a few days, when, if no further reduction took place, the whole was reduced with zinc and titrated with a standard solution of potassium permanganate. By this method great accuracy is obtained, as in such analyses there is a tendency to get too high results owing to the presence of organic matter. The reagents and utensils used were all proved to be free from iron. The spleens were treated in the same way, but the blood could not be washed out of them.
REMARKS

on

\section{OCULAR SYMPTOMS OF GENERAL PARALYSIS} OF THE INSANE, WITH SPECIAL REFERENCE T0 ITS CLINICAL GROUPINGS.

BY W. BEVAN LEWIS,

Medical Director of the West Riding Asylum, Wakefield.

\section{(Concluded from page 1025.)}

SECOND GROUP.

THIs embraces the subjects just referred to, being characterised specially by the occurrence of cycloplegic iridoplegia. The cases comprised in this category are sufficiently defined in their history, symptomatology, and course as to claim the position of a separate group.

(a) Mental Symptoms.-These, in an early stage, are marked by a light-hearted buoyancy arising from a general feeling of bien-être-a peculiarly self-satisfied and nonchalant air, which is associated with much playful gaiety and a careless, flippant manner betokening early dementia. In fact, such cases may be described as childish, good-humoured, and addicted to levity and pranks. Their egoism is occasionally indicated in their criticism of others (to their obvious disadvantage) associated with them-in their argumentative tone-love for a display of their own abilities, and the tendency to ridicule others. They are usually very careful in their attire, and their conduct throughout indicates selfadmiration and content. Memory is early implicated; attention is enfeebled, but early moral flaws are not so obvious as in other forms. They are talkative, jovial busybodies, fond of hoarding up, and almost invariably entertain exalted notions and ideas of great wealth and prosperity. Acute excitement is apt to occur usually in association with convulsive attacks which are present in a large proportion of such cases towards the end. In one notable case, acute aural and visual hallucinations were present for several months before the fatal termination.

(b) Specch Troubles.-Articulatory troubles, although a very notable feature in all these cases, was a later sequel to the oculo-motor. Thus in the case of . J. M. well-marked loss of both iridal reflexes and accommodation accompanied a very slight impairment of articulation, and this only perceived during the enunciation of difficult test words; whilst in $G$. H. B the enunciation was perfectly clear under all conditions. It is of interest to note that the thick, drunken, laboured, and hesitating utterance-the lips compressed to limit their tremulousness, and the resulting speech an indistinct muttering are the usual anomalies noted; and that where cases present disturbances of equilibration and ataxy, the speech is at times notably explosive, jerky, and forcible, so very different from the mumbling speech of the former. Both forms eventually approach utter unintelligibility.

In connection with the last-mentioned case, it is of interest to note that he presented at one time an instance of epileptiform seizures which could be induced whenever he was requested to protrude the tongue. I find on reference to my notes the following :

G. H. B., for the past six months, has been suffering from numerous convulsive seizures, and again last night he had a generalised convulsion. This morning he talks with a somewhat thick utterance but not muc apparent effort, and is quite clear mentally, recognising all that is said to him, and replying concisely and reasonably. Asked to protrude the tongue he fails to do so; evidently it costs him much effort. When urged to do so he partially succeeds, but a spasm immediately affects left side of cheek, and spreads into a genuine convulsive seizure limited to the face; the mouth was drawn to the left side, and the muscles of the face on this side were thrown into a state of violent tonic twitching. No convulsion of arm or leg occurred, and during the attack he drew the bedclothes over him with the left hand, talked freely and replied to questions, looked me straight in the face, and said, "I don't know you." Left side of face and ear hot and engorged from vasomotor paresis soles of feet exquisitely tender; plantar reflexiand cremasteric both in very great excess. Later on through the day whene

protrude the tongue left facial convulsions occurred. right side of the body with aphasia were twice induced by a $\frac{\text { strained mental effort. }{ }^{27} \text { This was also an instance of pro- }}{27 \text { Tescook of Mental Diseases, p. } 256 \text {. }}$ 
gressive paralysis of the insane, nor have I met with this ready explosiveness of nerve tissue in others than this disease.

(c) Spinal Symptoms.-These are not prominent features; in 2 cases the knee-jerks were defective or absent, but in the large majority of instances they were either unaffected or in excess. The gait was usually brisk, no stiffness of limbs being presented, and in all subjects death ensued without the development of contractures.

A large percentage of the individuals comprising this group give a clear history of syphilitic infection, and this at a period long antecedent to the nervous affection. The onset of nervous symptoms is essentially a rapid one, more especially when convulsive phenomena, which are here peculiarly frequent, usher in the decadence. Such resulting reductions are then of a very profound nature, induce an abrupt transition to the final dementia, and augur a rapidly fatal issue.

(d) Eye Symptoms.-I regard the eye symptoms as the earliest to appear in this group; they certainly antedate in several cases the disturbance of spinal reflexes. All the cases exhibited ocular anomalies upon admission, that is, well-marked associative iridoplegia, which sooner or later passed into cycloplegic form, the patient requiring plus glasses for reading with unimpaired distant vision :

J. M. $=+5$ D.; G. H. B. $=+5$ D.; A. D. $=+4$ D.; S. C. $=+4$ D.; W. R. $=+2.5$ D.; W. B. $=+2$ D.; T. G. $=+2.25$ D. The dilated pupils are paralysed, both as regards sympathetic and motor oculi supply, for not only do they fail to react to light and on convergence, but they do not respond by dilatation, either on shading or upon sensory stimulation. ${ }^{2 \theta}$ Co-ordinate movements are likewise incapable of exciting reaction, probably because the vasomotor of the iridal vascular supply is so completely involved. Eserine will contract such pupils ad minimum, and atropine dilate to the maximum extent.

To sum up the characteristics of this class :

(1) Early oculo-motor impairment-an associative iridoplegia, eventually with cycloplegia superadded.

(2) Early speech troubles of a profound nature ("drunken speech").

(3) Very infrequent spinal implication and no contractures.

(4) Notable egoism, self-assertion, and argumentative

(5) Very rapid course, the final result ushered in by severe convulsions and profound resulting reductions, both mental and physical.

Spasmodic or irritation mydriasis occurs, although infrequently, in progressive paralysis of the insane. It may also be induced by reflex agency, as indicated by von Brandenburg ${ }^{29}$ in a case where the light and convergence reflex were preserved, and also by the irritation of intestinal worms and other sources of irritation. ${ }^{30}$

The Cycloplegia. - The failure of accommodation was bilateral in all cases, and in two individuals only was the cycloplegia not associated with iridal implication; in fact, paralysis of the sphincter iridis as well as the sympathetic supply to the iris almost invariably accompanied the ciliary paralysis. The pupils were only exceptionally very large, and no response could be obtained to light, to shading, or to sensory stimulation. At the same time the associative iridoplegia was not only complete, but, like the accommodative failure, bilateral.

In view of the segmentation of the third nerve nucleus, now well established, and in reference especially to the functional significance of such segmented nuclei, ${ }^{31}$ it is open to suggestion that degeneration of these closely approximated centres of innervation might explain the cycloplegic iridoplegia in such subjects but for the serious difficulty already enunciated. It might be assumed that a lesion involving the median nucleus and the two pale club-shaped nuclei

28 Moeli has recognised this absence of reflex dilatation in general paralysis in 13 per cent. male subjects where the light reflex was preserved, and 65 per cent. males and 50 per cent. females where the light. reflex was abolished (Archivf. Psych., B. xiii).

29 Neurol. Centratbl., r893, p. 75r.

3 On the Segmentation of the Nucleus of the Third Cranial Nerve, by Alexander Bruce, Proc. Roy. Soc. Edin., vol. xvii
(Edinger-Westphal)-termed postero-internal by Bruce ${ }^{32}$ and the two anterior nuclei would explain the paralysis of the tensor choroideæ (cycloplegia) and the sphincter iridis, both for light and during convergence; but, since the pupils are fixed, failing to dilate also, we have still to reckon with the sympathetic, the vasomotor supply of which does not, so far as we at present know, come into relationship with these nuclei. So far as our present anatomical knowledge extends the ciliary ganglion is the only site at which the fibres for the tensor choroideæ, sphincter iridis, and dilating mechanism of the iris come into close apposition, and it is here, therefore, that Mr. Hutchinson points out that a lesion might be anticipated.

If this view be accepted we must be prepared to admit the occurrence in our cases of a bilateral and symmetrical implication of this small ophthalmic ganglion, whilst a further difficulty presents itself in the fact that we should a priori anticipate in this event a simultaneous affection of all its nerves ; whereas iridoplegia (that is, sympathetic and motor oculi) appears to precede the cycloplegia, and this creeping paralysis might seem more in consonance with a progressive degeneration of the anterior or postero-internal nuclei of the third nerve, eventually invading the median nucleus. It may, however, be regarded as certain that such a lesion as would affect the nuclei in the medulla and cause cycloplegic iridoplegia would (even in case of a proved connection betwixt these nuclei and the vasomotor) simply cut off the influence connected with varying degrees of light, and hence impair simply the dilatation of the pupil on shading; the dilatation upon cutaneous stimulation would still remain in force through the cilio-spinal tract. Now in several instances of cycloplegic iridoplegia this was not the case, for no dilatation occurred upon sensory stimulation; and I think it may be taken for granted that a large pupil with cycloplegic iridoplegia failing to respond both to sensory stimulation and to shading indicates implication of the ciliary ganglion, whilst if there be response to sensory stimulation and not to shading it indicates nuclear degeneration of the third nerve. The difficulty of accepting the ciliary ganglia as the site of lesion is fully acknowledged by Mr. Hutchinson: "One fact I must ask especial attention to ; it is that the paralysis of the iris appears to precede the loss of accommodation, and also to resist treatment longer." Again he says: "In none was the failure of accommodation the first symptom-a fact the more important when we consider that it causes a condition which the patient is not likely to overlook; whereas a motionless pupil may easily escape attention. It is, I admit, difficult to explain on the theory that the disease is in the ganglion why the ciliary muscle and the sphincter of the pupil should suffer in different degrees."

Cycloplegia without mydriasis (such as occurs after diphtheria) I have not met with, the pupils being moderate or very large, yet not a maximum mydriasis. In one case, however, of general paralysis in a female, paralysis of accommodation and of convergent contraction was present with very incomplete paralysis to the light reflex.

It has been already noted that in a large proportion ( 5 out of 12) constitutional syphilis was definitely ascertained, and quite in accordance is this with the history afforded by Mr. Hutchinson of his tabetic cases: "I cannot but regard it as highly probable that in almost all cases the affection is due to syphilis. We may note that it does not appear to occur amongst the later forms of tertiary disease, but rather during that period in which, after disappearance of secondary symptoms, there is usually a long period of latency."34

The rapid and acute development of cerebral symptoms with the severe convulsive seizures is a most striking feature in this group no less than the absence of spinal lesions ; possibly the descending changes in the cord and the resulting contractures are simply escaped owing to the very rapid course of this affection, the convulsions so quickly leading to a fatal issue.

Ophthalmoplegia externa is of very infrequent occurrence in genuine progressive paralysis of the insane, and when present is usually incomplete and associated with affection of the intrinsic muscles of the eye. Suckling records two 32 Op. cit.

33 Loc. cit.. p. 224

34 Ibid., p. 223. 
interesting cases, aged 21 and 18 respectively, where ptosis, strabismus, nystagmus, and greatly restricted movements of the eyeballs were associated with symptoms suggestive of general paralysis, but it is extremely doubtful whether the latter term should be used to indicate the functional disturbance in such cases. ${ }^{35}$

Siemerling describes a similar case of chronic ophthalmoplegia with progressive paralysis. ${ }^{35}$

\section{THIRd Group.}

We now come to a very important category of cases where the pupils tend to become small and spastic, and in all (with two exceptions) of the instances tabulated, myosis was present upon the patients first coming under observation and persisted throughout the affection. Eighteen cases in all presented this feature, and in 12 of these the knee-jerk was wholly abolished ; in 4 others it was recorded as sluggish so that in 2 cases only out of the 18 was the knee-jerk norma or in excess. It may also be noted that as many as I I showed grave defect of equilibration by the Romberg test, and that ro presented beyond this static ataxy, a more or less serious ataxy of locomotion; manual ataxia occurred in 2 subjects only.

Here then we have an association of symptoms closely resembling those of ordinary tabes, namely : myotic pupils, abolished knee-jerk, failure of equilibration, and locomotor inco-ordination. In 4 only of these 18 cases could it be said that as in typical tabes cutaneous sensibility was notably impaired; 2 of these presenting also lightning pains, as did one other where no cutaneous anæsthesia was observed.

As to the mental disturbance, depression was not a prevalent or even noticeable feature in these cases. Much more frequent was the association of excitement with optimism, in some instances rising to extremes of the grandiose, with very profound mental enfeeblement or dementia. In several of these cases thievish propensities were very notable features. In connection with cortical implication it is important to note that two cases alone suffered from convulsive seizures.

Oculo-Motor and Spinal Implication.-In almost every instance the accommodative movements of the iris were preserved-the Argyll-Robertson sign prevailing here as it does in a group still to be noted, and characterised by a spastic paraplegia. Two cases forming the only exception-one of these being, moreover, a good illustration of the tabetic arthropathy described by Charcot and involving both knees. The case of J. M. may be taken as typical of the class. The pupils were very small, equal in size, fixed to light, but not to convergence; there was loss of knee-jerk, the Romberg sign existed with well-marked locomotor ataxy, slight manual ataxy, ordinary sensibility intact, superficial reflexes unimpaired, and characteristic Charcot's joints. It is of interest here to note that our cases afford a decided answer to the question, which sign first presents itself in order of timethe loss of myotatic irritability expressed in impaired or abolished knee-jerk, or the oculo-motor implication? In four patients a sluggish response could still be elicited on percussing the patella tendon, although reflex iridoplegia was notably present in both eyes; whilst still more to the point are the cases of three others, where the knee-jerks were even in excess, with well-marked Argyll-Robertson pupils-becoming, however, lost later on in the course of the disease. In these tabetic forms of general paralysis we may fairly regard ithe initial symptom, therefore, as usually, if not invariably, oculo-motor-the loss of the knee-jerk appearing later on.

The cilio-spinal implication was attested to in the complete failure to elicit dilatation either by shading the eyes, by cutaneous stimulation, or by co-ordinate movements of the limbs. It must be noted also that the order of implication in these cases could not be definitely decided as a myosis followed by reflex iridoplegia-that is, a paralysing affection of the vasomotor (cilio-spinal region), ascending to the quadrigeminal level, and eventually implicating the motor oculi constrictor fibres. In all cases alike the small myotic pupil was found from the first associated with the absence of light reflex.

s5 Functional Ophthalmoplegia with General Paralysis and Implication of Cranial Nerves in Young Women, by C. W. Suckling, M.D., BRITISH MEDICAL JOURNAL, r803. p. 634

$$
{ }^{86} \text { Neurol. Centralbl, } 1893, \text { pi } 253 .
$$

It is now fully admitted that in tabes dorsalis cases occasionally occur where myosis is not a notable feature, where the pupils are of moderate size or even somewhat dilated, and yet exhibit the Argyll-Robertson sign (Raehlmann) ${ }^{37}$ Again, in lesion of the cervical sympathetic, although the usual statement made is that the pupil on the affected side is contracted; yet many exceptions occur to this, and Mr. Hutchinson states that his own observations lead to the belief that not contraction, but simply inability to dilate, is the usual feature: "I have examined the state of the pupil in six examples of paralysis of the cervical trunk of the vasomotor, and in none was its size ever less than about No. 4 (pupillometer $=3 \mathrm{~mm}$.)" 38

It is, however, important to regard the two indications as wholly distinct and of regional significance, the myosis indicating disease of the posterior columns of the cord, the loss of light reflex, with retention of the associated contraction on convergence, indicating disease of the optic nerve fibres, or their connection with the nucleus of the third

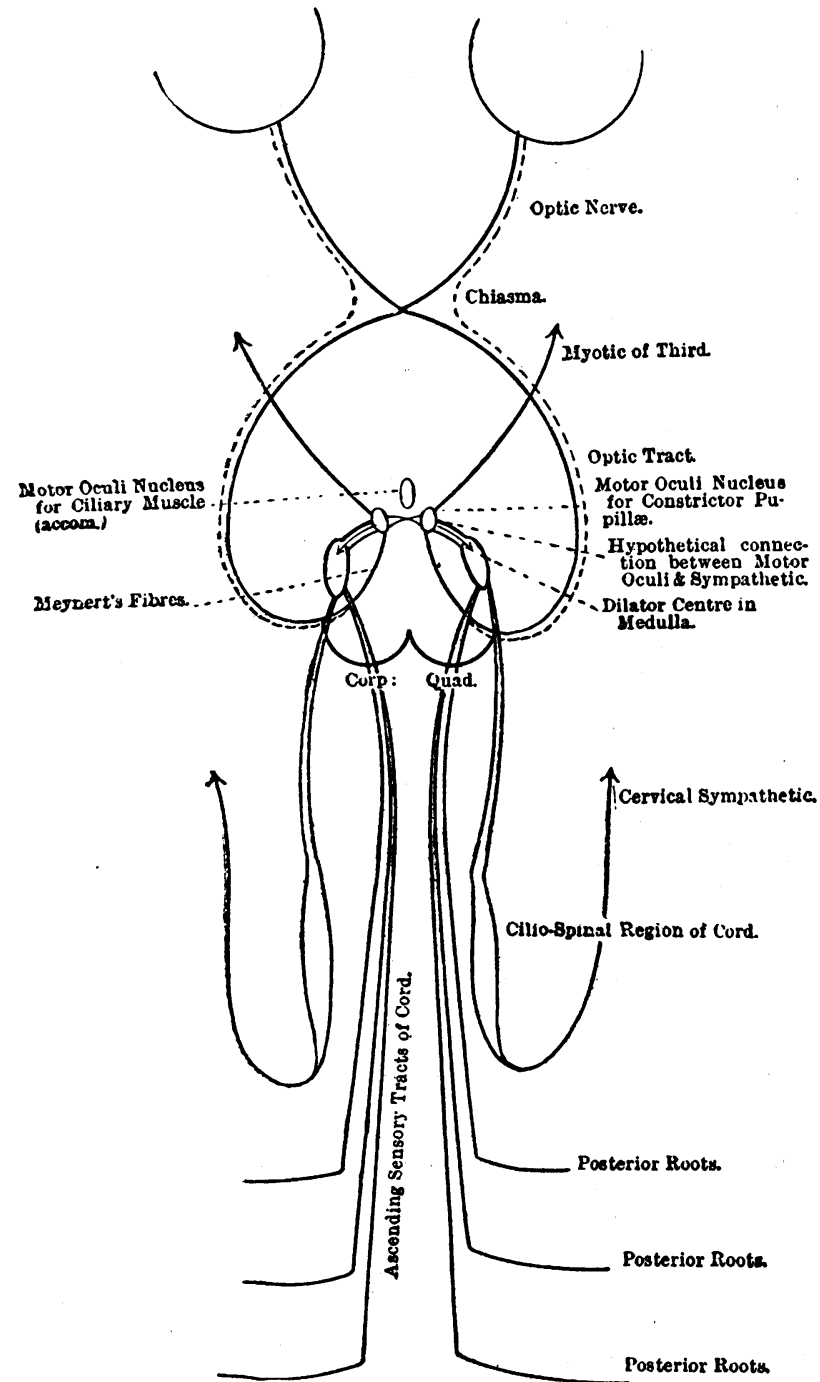

nerve, or Meynert's fibres (Swanzy). ${ }^{39}$ We have seen reason, however, for regarding this explanation with doubt; in bilateral affections of Meynert's fibres such, doubtless, would be the symptoms ; but in unilateral lesions of this connecting tract no effect on the light reflexes would ensue owing to the ${ }^{37}$ Archiv f. Physiologie, 1878.

${ }^{38}$ Notes on the Symptom Significance of Different States of the Pupil Brain, vol. i, p. rr.

39 Diseases of the Eye, 1890, p. $275^{\circ}$ 
existence of a commissural tie betwixt the two motor oculi nuclei. Moreover, we know that these conditions are often unilateral, for example, in two illustrative cases, where we see with left reflex iridoplegia, associative movements being intact, the sympathetic reflex could not be obtained; sensibility, however, was notably defective.

Our only resource, therefore, is to seek an explanation for the defective reaction in the nucleus itself, and this is fully authorised by the knowledge of its segmentation now admitted.

All further difficulty disappears if we stippose that fibres directly connect the "constrictor nucleus" with the vaso-motor tract in the medulla. Such connecting fibres would have to be regarded as inhibitory, their excitation through optic stimuli inhibiting the normal action of the cilio-spinal tract. Shading the eyes (in normal subjects) would reduce such activity and favour dilatation : division of such fibres would, of course, act likewise, whilst disease lower down in the ciliospinal region would induce a myosis through the unrestrained activity of the motor oculi.

Such moderate myosis as is usually seen in disease of the cervical sympathetic is not what we find in our cases of tabetic general paralysis; the pupil is usually very smallfrom I mm. to 2 or $2.5 \mathrm{~mm}$. at most, and would appear to be due to a lesion advancing upon the "constrictor" nucleus of the third nerve, introducing by its proximity an element of irritation, the paralytic myosis being now transformed into a spastic myosis. Contraction upon convergence is still retained, the light reflex is lost, whilst the myosis and failure to dilate upon shading or sensory stimulation indicates the implication of the cilio-spinal region also.

Should these (hypothetical) inhibitory fibres alone be implicated, a moderate or even large pupil might be seen which fails to dilate on shading, but does so on cutaneous stimulation, whilst the reaction to light might be more or less sluggish or impaired; if the disease extends up towards the "constrictor nucleus" itself, the Argyll-Robertson sign would supplement this condition with spastic pupil becoming large when the nucleus is destroyed. On the other hand, if the cilio-spinal or cervical sympathetic be diseased, the myosis would be associated with failure to dilate on shading, and with all forms of sensory stimulation.

\section{Fourth Group.}

As best illustrative of this series, I may briefly record two cases typical of the group.

J. H., a dyer, aged ${ }_{36}$, married, and has a family of two children, was all the attendant restessness and incoherent irratiacal excitement, with was atident reslessness and incohent was considerable reduction in consciousness, entire loss of adaptation to his surroundings; mood variable; cheerful, careless laughter, interspersed with causeless sobbing and fretfulness; grandiose notions, not prevalent, but a certain amount of egoism betrayed by his references to awkwardly, constantly varying his position, and "looking behind him as if awkwardly, constantly varying his position, and "looking behind him as if expecting to see something. Mental and articulatory troubles had been noted twelve months prior to admission. Hereditary insanity, personal evidence of syphilis forthcoming. He was of middle height, thin, reduced, evidence of syphilis forthcoming. He was of middle height, thin, reduced,
very feeble and tottering. His gait was sprawling, uncertain, distinctly very feeble and tottering. His gait was sprawling, uncertain, distinctly
ataxic, and requiring a wide basis for support; would certainly fall if the ataxic, and requiring a wide basis for support ; would certainly fall if the paired, the muscles lax and flabby. Knee-jerk was in considerable parces, the muscles lax and flabby. Knee-jerk was in considerable excess; no ankle clonus, and plantar reflex was normal. His speech was tremor, its protrusion effected with considerable effort, much contortion tremor, its protrusion effected with considerable effort, much contortion and grimace. The pupils were both widely dilated, and reacted sluggishly was present. A week after admission it was noted that he frequently was present. A week after admission it was noted that he frequently
turned round from left to right. the gyration being preceded by turned round from left to right, the gyration being preceded by his limbs, but suffered no vertign: these movements were usually initiated when he attempted to walk along a straight line. The right pupil was the larger of the two; speech was greatly slurred.

Three months after admission was extremely emotional and profoundly demented; bulbar symptoms still more marked and muscular enfeeblement greater; gait notably insecure and ataxic; less tendency to gyrate.

Nine months after admission pupillary symptoms were: Reflex iridoplegia with mydriasis, the right being the larger. At this time a slight paretic seizure occurred on right half of body, leaving right arm and leg somewhat stiffened. Knee-jerks remain exaggerated; clonus in both ankles, and plantar reflex also in excess.

No further "seizures" occurred. The right arm remained slightly stiff; the fingers closed upon the palm, with slight vasomotor flushing and some œedema of integument, and he died from exhaustion two years and seven montlis after admission.

In this case we note maniacal reductions with slight egoism, but no notable grandiose or optimistic state; special difficulties for twelve months prior to admission, followed by ataxia (locomotor) and advancing enfeeblement. The awkwardness of pose - the turning of the head as though looking for something behind him described by the friends was certainly of the same nature as the tendency to gyrate round his axis from left to right. Then upon admission we recognise notably the Romberg sign-the locomotor ataxy, the great excess of knee-jerk, advanced muscular enfeeblement, and param lytic mydriasis (Argyll-Robertson form) with unequal pupils. H. S., aged 54, married, and has three children. Patient is a carter, and was admitted in September, x891, the duration of mental ailment stated state was one of mild dementia-a sluggish apprehension and a tardy laboured response. He had delusions of suspicion with reference to his wife; is distrustful of her; says she has driven nails into the outer walls of the house for men to attach ropes to and so enter the house at night: has seen their footprints on the floor, and hears their footsteps down stairs. If the doors and windows are closed le still thinks men get in through the wall behind the fireplace, and he swears at people whom he hears passing the door. Hitherto there had been no grandiose notions. For six months he has found difficulty in walking, and has suffered from vertigo to such an extent as to be unable to follow his occupation; for four months he has entertained these suspicions against his wife, these having been ushered in by an epileptiform seizure. Excitement followed for twenty years patient has been a heavy drinker.

On admission, speech drawling, "drunken," at times almost uninterligible, as though his mouth was full of food ; no stammering or hesitation. He is conscious of the defect; dates it back six months, at which time he also felt unsteady on his legs as if drunk when getting up in the morning; says his legs "wouldn't work regularly." Hands were also enfeebled in grasp, and pains - "not severe but very sudden and quickly tions of bladder and bowel norms and his knees. No headache. Funcsystole at base; apex beat outside nipple line. Pupils were equal, reactions unimpaired: tongue protruded with considerable effort; no fibrillax tremor; slight twitching of facial muscles; knee-jerk excessive; tap followed by universal clonic movements of whole leg. Clonus notable and prolonged in both ankles. Plantar reflex excessive. No paradoxical of locality defective in legs only. General sensibility defective in lowen. extremities, but not temperature sense. Slight delay in transmission of extremities, but not temperature sense. Slight delay in transmission of
sensory current. He takes a broad basis of support, sways much, foot sensory current. He takes a broad basis of support, sways much, foot
stiff and held at right angles to leg, comes down heavily upon his heels ; stiff and held at right angles to leg, comes down heavily upon his heels; the legs are jerked forwards, and he is most unsafe in turning round? up canjects from the floor: no evident loss of power in the leg muscles ; up objects from the floor; no evident loss of power in the leg muscles;: no wasting and no atonicity. No manual or trunk ataxy, and no tremors of the limbs. Dynamometer = right 7o kilos., left 50 kilos. Vision was good; no achromatopsia; eyes well

In this last case, therefore, after twenty years' hard drinking, we see the development of locomotor ataxy with lightning pains, speech troubles, vertiginous seizures, six months prior to his admission, followed two months later by an epileptiform seizure ushering in mental symptoms of the nature of acute auditory hallucination, delusions of suspicion in his marital relationships, and advancing dementia. Upon. admission speech almost unintelligible; sensibility and locomotion defective, yet no eye symptoms perceived. The spinar symptoms are still more emphasised than in the first case;: the spinal troubles also more advanced. In several other cases of alike character the pupillary failure did not precede, but followed upon, the full development of ataxia, the advance, in fact, being stated as follows: Spinal troubles (ataxic paraplegia); speech troubles, very notable ; mentar troubles after epileptiform seizure ; eye symptoms last of all. Of the six cases belonging to this category, four presented spinal symptoms earlier than eye symptoms, and in the other two both symptoms were present on admission, their rear sequence not ascertained. In all the cases except that of H. S. sensibility remained unaffected, and ataxy with enfeeblement and myotatic irritability were throughout restricted to the lower extremities. Gowers refers to slight articulatory impairment as not uncommon in typical cases of ataxic paraplegia and "irregular tremulous movements of the face, resembling those of general paralysis, and this in cases in which there is no mental change" ${ }_{40}$; he states, moreover, that as a rule in such cases, "the mental state is either normal, or there is merely slight failure of memory." In such cases, of course, it appears highly desirable that $a$ careful examination of the cerebral cortex and membranes should be made ere we exclude them from the category of general paralysis. ${ }^{41}$

40 Diseases of the Nervous System, vol. i, p. 343

41 The association with general paralysis is fully admitted by Gowers, who says: "Amongst complicetions the most important are mental. indeed this combined sclerosis may form part." 
In contrast with typical cases of spinal ataxic paraplegia these instances therefore chiefly differ in :

(1) The non-extension of myotatic irritability to the arms.

(2) The almost invariable iridal paralyses.

(3) The extreme speech difficulties and ataxic movements of the tongue and facial muscles.

(4) The pronounced mental derangements.

In one case only did lightning pains present themselves, and in this respect the spinal symptoms were similar in both typical and general paralytic cases. I have elsewhere ${ }^{42}$ recorded a well-marked instance in the female of ataxic paraplegia with general paralysis, in which the small myotic pupil becime dilated-where ataxia and profound paralysis affected both upper and lower extremities-and where successive attacks of right hemiplegia with hemianæsthesia and aphasia were eventually followed by almost universal contracture of limbs before death. In this case the mental features notably pronounced, were delirious excitement with grandiose states. In fact, in most of the cases observed, acute excitement prevailed as the initial stage, and later on, very profound dementia. In contrast with this profound mental enfeeblement it is instructive to compare a case of simple ataxic paraplegia recorded by Dr. Michell Clarke, in which the lesions found were exclusively confined to medulla and cord, the brain being free from any obvious implication. ${ }^{43}$

It is not to be inferred here that the arms invariably esc ipe from inco-ordination, but that a definite systemdis zase productive of ataxic paraplegia characterises this group. The occurrence of multiple sclerosis, which is not unusual in general paralysis, may of course complicate any one or other of the forms described as illustrated by the following brief notes of a case:

D. W. is very optimistic and grandiose, possesses $£ 10,000$ and any amount of landed property. Much nocturnal restlessness and excitement. There is considerable loss of power in the lower limbs. Notable spastic gait, but no Romberg sign or locomotor ataxy. Knee-jerks both in large excess : slight ankle-clonus on both sides; paralysis of bowel and bladder. Extreme ataxy of left arm with continuous sclerotic tremor of the hand, especially when the right is occupied, as in feeding; utter inability to use left hand for any purpose; much tremor of both arms, especially the left upon extension; myotatic excess on percussion of triceps and supinator. Tongue shows extreme ataxic jerkiness. Extreme sensitiveness to pricking or tickling the skin ; much timidity displayed. Pupils small, equal, normal in reaction; no impairment of vision, no nystagmus.

\section{Fifth Group.}

Upon eliminating from the total number of cases observed all such as were comprised under one or other of the categories already described, we find a residual number (23 in all) characterised by the predominance of mental symptoms and the absence of the more striking physical evidences of typical cases of general paralysis. Thus, inequality of pupil, though occasionally present, is of most infrequent occurrence, whilst the iridal reflexes are invariably intact; the pupil may be in some cases small in others dilated, but in a much larger proportion of cases is it of moderate dimensions. The knee-jerk, occasionally normal, is more frequently in great excess, together with ankle clonus; but, apart from this, spinal symptoms are remarkable for their absence. Thus, equilibration and coordinate movement of the limbs is maintained throughout, until advancing debility confines the patient to bed; no disturbance of general sensibility is perceived, and the gait, at first brisk and firm, presents at no later stage indications of an ataxic paraplegia on the one hand or of a spastic enfeeblement on the other. Such cases are-like those of the third group-notable for the comparative rarity of convulsive seizures which in the other forms lead to such rapid and serious reductions; neither is the tremor of tongue and facial muscles so marked a feature as in other groups of the disease; grinding of the teeth is an occasional symptom, whilst severe cephalagia is often complained of.

Although the sudden and severe reductions following upon 42 Mental Diseases, pp. 280-283.

3n a Case of Ataxic Paraplegia with Autopsy, letc., by J. Michell 7 the seizures of the convulsive forms do not distinguish this group, yet profound reductions do occur at a very early stage of the illness; in fact, most of these subjects present advanced dementia upon their admission to the asylum. Optimism with noisy excitement, destructive tendencies, and degraded habits are notably absent, although their nocturnal reductions may be attended by occasional noisy, maniacal outbursts. From the first, however, the chief features recognised by the friends have been a dull, heavy, stuporose state, with advancing mental enfeeblement. Such cases merit the designation of a primary progressive dementia, and suggest the more or less complete escape of the central gyri from implication. As a fact, it may be stated here that this is the case, for adhesions of the pia arachnoid are extremely slight or entirely absent in such cases in the motor realms. In 75 per cent. no adhesions were present on any portion of the motor cortex, and adhesions were com pletely absent in 44 per cent. from all parts of the brain alike When present such adhesions usually occupied the precentral region, the three frontal gyri being in such cases much attenuated; or they spread over the inferior and outer aspects of the hemisphere, the temporo-sphenoidal, supramarginal, or angular gyri, or, again, the mesial aspect of the cerebrum. In one case, however, firm adhesions occurred over the lower part of the right central gyri ; and in this instance continuous grinding of the teeth was a most notable feature, together with convulsive seizures. As regards this symptom of cerebral irritation, I have observed that when the right hemisphere in the neighbourhood of the third frontal and ascending frontal convolutions are much involved by adhesions of the investing membranes, grinding of the teeth is of much less frequent occurrence than in similar affections of Broca's and the adjacent gyri of the left hemisphere. This symptom, often painfully distressing to others, can in these subjects be almost immediately relieved by the administration of a very small dose of chloral (5 grs.), a treatment I always strongly advo zate.

It appears to me that in the ensemble of symptoms here depicted we have full justification for the establishment of a distinct group framed upon clinical and pathological basis. Throughout the whole course of the disease the cortical areas which, when injured, lead to descending changes in the medulla and spinal cord, escape implication here; throughout the whole history of the case convulsions (which predominate so largely in other forms where the motor cortex is affected) are particularly infrequent, and neither sympathetic nor motor oculi supply to the iris suffer at any time. A primary dementia has been noted by others as characterising a certain number of cases. Thus Mickle ${ }^{44}$ writes :

In a few cases of general paralysis a dementia begins, progresses, and practically includes or conditionates the entire range of mental symptoms throughout the whole course. It is a question whether these are not examples of general paralysis in its most pure and simple form. Although embraced under the present head, these are not more referred to here than are the cases in which dementia, although the predominant symptom, is associated with other mental phenomena, such, indeed, as may occur in other forms of dementia.

I cannot help thinking that such cases as are here described by Mickle include the subjects I would embrace in my fourth group, but no mention is made in connection with these cases of the notable absence of physical accompaniments to which I have referred. It is possible, however, that his second group described at page $409^{45}$ may be identified with the series here described. ${ }^{46}$

In conclusion, for convenience of reference, I will here tabulate those features which mainly demarcate the five groups usually included under the category of general or progressive paralysis of the insane.

GrouP I.

Paralytic mydriasis ; a partial reflex iridoplegia (light).

Increased myotatic irritability.

Excessive facial tremor and speech troubles.

Great optimism with profound dementia.

Group 2.

Mydriasis with associative iridoplegia rapidly passing into the cycloplegic form - an early symptom.

44 General Paralysis of the Insane, and edition, p. $3^{\circ}$. 45 Op. cit.

46 See also in this ronnection a suggestive paper "A Variation of Type in General Paralysis" by F. St. John Bullen, Journal of Mental Science, 1893. p. 185 . 
Frequent myotatic excess but no contractures.

Late speech troubles.

Acute excitement with frequent convulsions.

Very rapidly fatal course. (Preponderance of syphilitic history.)

Group 3.

Spastic myosis; a complete reflex iridoplegia.

Absent or greatly impaired knee-jerk.

Failure of equilibration; locomotor ataxy, defective sensi-

bility.

Very defective articulation.

Much optimism and excitement.

Group 4.

Late eye symptoms: paralytic mydriasis, a partial reflex iridoplegia (for light only)

Ataxic paraplegia confined to lower extremities. (Arms do not participate.)

Great facial ataxy with extreme troubles of speech.

Epileptiform seizures ushering in pronounced mental enfeeblement.

GroUP 5 .

No oculo-motor symptoms beyond occasional inequality.

No contractures, but notable myotatic excess.

No disturbance of equilibration, locomotion, or sensation.

Speech troubles not pronounced.

Epileptiform seizures very rare, but from the first progressive deepening dementia.

\section{VERTEBRAL PUNCTURE IN GENERAL PARALYSIS OF THE INSANE,}

WTTH SOME REMARKS ON THE PRESSURE AND COMPOSITION OF THE CEREBRO-SPINAL FLUID IN THIS DISEASE.

BY JOHN TURINR, M.B.ABERD., Senior Assistant Medical Officer, Essex County Asylum.

Is 1889 Dr. Claye Shaw ${ }^{1}$ trephined in a case of general paralysis, and shortly afterwards Dr. Batty Tuke reported cases on which he had operated by trephining, and strenuously upheld the new departure.

The normal pressure of the cerebro-spinal fluid in man is stated by Leyden ${ }^{3}$ to be equal to a column of water ro to 12 centimetres high. But this is very probably somewhat underestimated, as Dr. Dean, experimenting with dogs weighing from 30 to $60 \mathrm{lbs}$. found that in them the normal pressure varied between 7 and 15 centimetres of water. ${ }^{4}$ Foster (p. I129) gives the pressure in dogs as equal to io to II millimetres of mereury. Later on it will be seen that rarely in the cases here recorded was the pressure in excess of that found by Dr. Dean in his larger dogs.

During the last year or two very little has been heard of trephining in general paralysis. The operation at first excited considerable discussion; those who looked upon the excess of fluid in this disease as compensatory could see no object in: the procedure; but, nevertheless, if beneficial results followed as they did, for a time at least in most of the cases, these results would have to override any speculative views as to the nature of the fluid. 'It was on this account, although believing that the fluid was compensatory, that I undertook to test the effects of lumbar puncture in general paralysis. The operation is simple, is apparently in most cases unattended with danger, and most certainly will relieve pressure in the cranium ; it thus fulfils, at least temporarily, all the conditions that result from trephining, or the more elaborate operation of laminectomy.

Vertebral puncture was, I believe, introduced by von Ziemssen for the relief of pressure in cerebro-spinal meningitis, cerebral tumour, hydrocephalus, etc., and various notes respecting it occur in the EPITOME of the BRITISH MEDICAL JoURNAL from 1893 to the present date. One important statement in reference to the subject here treated is made by von

1 BRITISH MEDical JodRnal. June I4th, 1890.

2 BRITISH MEDICAL JoURNAL, January $16 \mathrm{th}$, 1892

3 Quoted by Drs. Macpherson and Wallace, BRITISH MEdical Journat, and

4 Jour. of Path. and Bact., vol. i, pp. 30-3т.
Ziemssen, ${ }^{5}$ that in cases where the pressure is high the fluid issues in a fine jet from the trochar; where it is low it comes away in drops.

I have performed the operation on 14 cases of general paralysis-7 maies and 7 females; and, speaking broadly, I may say that it has not been followed by any appreciable amelioration in the condition of the patient either in the bodily or the mental aspects of the case. One woman, who was usually very noisy at night, was quiet for a few nights after the puncture, and slept better, but became noisy again after a few days. She was punctured a second time, six weeks later, and again became quiet at night, but the only other effect produced was, according to her own account at the time of puncture, "a splitting headache." I have noticed no alteration either in the size or the reaction to light of the pupils afterwards. Nor is the speech in any way improved. One patient (No. XIV in the list) was punctured on February $22 n d$, at 2.30 P.Mand $44 \mathrm{c} . \mathrm{cm}$. of fluid withdrawn. The following day, at 3 P.M. he had a succession of strong convulsive seizures lasting with short intervals for more than an hour, and affecting his left side, during which his temperature rose to $102.8^{\circ} \mathrm{F}$. This of itself would seem to negative the idea that the symptoms in the seizures were due to any excess of pressure. All the cases punctured were between the ages of 3 and $_{57}$. The majority (Nos. I, II III, IV, tured were between the ages of $3^{\text {I }}$ and 57 . The majority (Nos. I, II, III, IV, v, VII, XI, XII, and XIII), aithough in an advanced stage of the disease, were in good bodily condition, and still possessed considerable intelligence and memory. They had all had seizures. In Nos. XI and XIII the just over one year in No. XI and only six months in XIII. Both had the just over one year in No. XI and only six months in XIII. Both had the characteristic grandiose delusions of general paralysis, and had been engaged at their respective occupations almost up to the time of their admission to the asylum. The remaining

Now although from a therapeutic point of view vertebral puncture must be regarded as a failure, yet the facts obtained by noting the pressure in each case as well as from the subsequent examination of the fluid are full of interest. They are directly opposed, on the one hand, to the idea that the excess of fluid in general paralysis is pressure fluid, and, on the other, to the idea that this fluid is an inflammatory product. They show that in the cases examined, with perhaps two exceptions, there was no pressure present beyond that likely to belong to the blood pressure, and that the nature of the fluid, as regard at least its proteid constituents, did not differ from normal cerebro-spinal fluid, and that it had none of the characters of an inflammatory exudation.

Pressure.

The procedure in puncturing was as follows: The patient was placed on his right side with his legs drawn up, his head was either supported on a low pillow (Nos. III, v, VI, VII, IX, and $x$ ), or else rested directly on the mattress. A fine hollow needle about 4 inches long was inserted a little to one side of the middle line between the second and third lumbar spines, and in a direction slightly upwards and towards the middle line. A long glass tube, bent near one end at right angles and marked on its long portion in centimetres (the diameter of the tube was 4 millimetres), was arranged so that its bent end could easily be connected with the needle as soon as the dura was punctured, which was shown by the clear fluid welling slowly out from the end of the cannula. In every case it came away in drops, not in a continuous stream or fine jet, as von Ziemssen observed it to do in cases where the pressure was high. Thus the tube could be connected with the needle without losing more than a drop or two of the fluid. On connecting the glass tube with the needle, and holding the long arm of it vertically, the fluid rose in it, and in some cases presented well marked but slight movements synchronous with the pulse. This was observed only, however, in three of the cases (Nos. IV, IX, XI); each of these had recently had a seizure; No. Ix a transient convulsive seizure the day before, and this man was, as subsequently determined, in the initial stage of an attack of pneumonia from which he died four days after. During the operation his temperature was $100.2^{\circ} \mathrm{F}$., rising in the evening to $103.6^{\circ}$. No. IV an apoplectiform seizure the day before, and No. XI a strong convulsive seizure five days previously. No. XII, in whose case the pressure was the highest recorded, had had an apoplectiform seizure three days before.

In every case talking and screaming raised the pressure, and with one woman (No. I), who at my suggestion took two or three inspirations, the fluid rose from 14 to 19 centimetres, and remained at the latter level all the time she held her breath. I mention this as Dr. Dean states in his paper (v.8., p. 29) that with inspiration the pressure falls. Pas-

EPI'TOME, May 27tli, 1893" 